\title{
Tigliane Diterpenoids from the Stem Bark of Neoboutonia macrocalyx
}

Peter G. Kirira, ${ }^{\dagger, \grave{ }}$ Geoffrey M. Rukunga, ${ }^{\ddagger}$ Alphonse W. Wanyonyi, ${ }^{\dagger}$ Charles N. Muthaura, ${ }^{\ddagger}$ Geoffrey M. Mungai,${ }^{\S}$ Alex K. Machocho, ${ }^{\dagger}$ and Isaiah O. Ndiege ${ }^{*}$,

Department of Chemistry, School of Pure and Applied Sciences, Kenyatta University, P. O. Box 43844, Nairobi 00100, Kenya, Center for Traditional Medicine and Drug Research, Kenya Medical Research Institute, P. O. Box 54840, Nairobi 00200, Kenya, and East Africa Herbarium, National Museums of Kenya, P. O. Box 40658, Nairobi 00100, Kenya.

*Corresponding author. Tel: +254-20-810901, +254-20-811622. Fax: +254-20-811575. E-mail address: indiege@icipe.org.

${ }^{\dagger}$ Kenyatta University.

${ }^{\dagger}$ Kenya Medical Research Institute.

${ }^{\S}$ East Africa Herbarium. 


\section{Supporting Information}

S1: 2D NMR data for Tigliane Diterpenoids (2-4)

\begin{tabular}{|c|c|c|c|c|c|c|}
\hline & \multicolumn{2}{|c|}{2} & \multicolumn{2}{|c|}{3} & \multicolumn{2}{|c|}{4} \\
\hline Position & ${ }^{1} \mathrm{H}^{-1}{ }^{1} \mathrm{H} \mathrm{COSY}$ & $\mathrm{HMBC}(\mathrm{C} \rightarrow \mathrm{H})$ & ${ }^{1} \mathrm{H}-{ }^{1} \mathrm{H} \mathrm{COSY}$ & $\mathrm{HMBC}(\mathrm{C} \rightarrow \mathrm{H})$ & ${ }^{1} \mathrm{H}^{-1} \mathrm{H} \mathrm{COSY}$ & $\mathrm{HMBC}(\mathrm{C} \rightarrow \mathrm{H})$ \\
\hline 1 & & $3,4,9,19$ & & $3,4,9,19$ & & $3,4,19$ \\
\hline 2 & & & & & & \\
\hline 3 & & & & & & \\
\hline 4 & & & & & & \\
\hline $5 \mathrm{a}$ & $\mathrm{OH}$ & $3,7,10,20$ & $5 \mathrm{~b}$ & $3,7,10,20$ & $5 \mathrm{~b}$ & $3,7,10,20$ \\
\hline $5 b$ & & & $5 \mathrm{a}$ & $3,7,10,20$ & $5 \mathrm{a}$ & $3,7,10,20$ \\
\hline 6 & & & & & & \\
\hline 7 & 8 & $5,9,14,20$ & 8 & $5,9,14,20$ & 8 & $5,9,14,20$ \\
\hline 8 & 7,14 & $6,10,11,13,15$ & 7,14 & $6,10,11,13,15$ & 7,14 & $6,10,11,13,15$ \\
\hline 9 & & & & & & \\
\hline 10 & & $2,3,5,8,11$ & & $2,3,5,8,11$ & & $2,3,5,8,11$ \\
\hline 11 & $18,12 \mathrm{a}, 12 \mathrm{~b}$ & $8,10,13$ & $12 \mathrm{a}, 12 \mathrm{~b}, 18$ & $8,10,13$ & $12 \mathrm{a}, 12 \mathrm{~b}, 18$ & $8,10,13$ \\
\hline $12 \mathrm{a}$ & $11,12 b$ & $9,14,15,18$ & $11,12 \mathrm{~b}$ & $9,14,15,18$ & $11,12 b$ & $9,14,15,18$ \\
\hline $12 \mathrm{~b}$ & $11,12 \mathrm{a}$ & $9,14,15,18$ & $11,12 \mathrm{a}$ & $9,14,15,18$ & $11,12 \mathrm{a}$ & $9,14,15,18$ \\
\hline 13 & & & & & & \\
\hline 14 & 8 & $7,9,12,16,17$ & 8 & $7,9,12,16,17$ & 8 & $7,9,12,16,17$ \\
\hline 15 & & & & & & \\
\hline 16 & & $13,14,17$ & & $13,14,17$ & & $13,14,17$ \\
\hline 17 & & $13,14,16$ & & $13,14,16$ & & $13,14,16$ \\
\hline 18 & 11 & 9,12 & 11 & 9,12 & 11 & $9,11,12$ \\
\hline 19 & & 1,3 & & 1,3 & & 1,3 \\
\hline $20 \mathrm{a}$ & $20 \mathrm{~b}$ & $5,6,7,1^{\prime \prime}$ & $20 \mathrm{~b}$ & 5,7 & & $5,7,1^{\prime \prime}$ \\
\hline $20 \mathrm{~b}$ & $20 \mathrm{a}$ & $5,6,7,1^{\prime \prime}$ & $20 \mathrm{a}$ & 5,7 & & $5,7,1^{\prime}$ \\
\hline $1^{\prime}$ & & & & & & \\
\hline $2^{\prime}$ & $3^{\prime}$ & $4^{\prime}$ & $3^{\prime}$ & $4^{\prime}$ & $3^{\prime}$ & $4^{\prime}$ \\
\hline $3^{\prime}$ & $2^{\prime}, 4^{\prime}$ & $1^{\prime}, 5^{\prime}$ & $2^{\prime}, 4^{\prime}$ & $1^{\prime}, 5^{\prime}$ & $2^{\prime}, 4^{\prime}$ & $1^{\prime}, 5^{\prime}$ \\
\hline $15^{\prime}$ & $a$ & $13^{\prime}$ & $14^{\prime}$ & $13^{\prime}$ & $a$ & $13^{\prime}$ \\
\hline $16^{\prime}$ & $a$ & $14^{\prime}$ & & & $15^{\prime}$ & $14^{\prime}$ \\
\hline 1" & & & & & & \\
\hline 2" & 3" & $20,4 "$ & & & 3" & $20,4 "$ \\
\hline 3" & 2", 4" & 1", 5" & & & 2", 4" & 1", 5" \\
\hline 4" & $a$ & 2", 6" & & & $a$ & $2 ", 6 "$ \\
\hline $11 "$ & $a$ & 9" & & & $a$ & 9" \\
\hline $12 "$ & $a$ & $10 "$ & & & $\mathrm{H}-11 "$ & $10 "$ \\
\hline
\end{tabular}

${ }^{a}$ Complete connectivity not determined due to overlap of ${ }^{1} \mathrm{H}$ NMR signals in this range. 